\title{
A Distributed Passivity Approach to AUV Teams Control in Cooperating Potential Games. «
}

\author{
Filippo Fabiani*, Davide Fenucci, Andrea Caiti \\ Department of Information Engineering, University of Pisa, Largo L. Lazzarino, 1, 56122, Pisa, Italy \\ Research Center “E. Piaggio”, University of Pisa, Largo L. Lazzarino, 1, 56122, Pisa, Italy \\ Interuniversity Center for Integrated Systems for Marine Environment (ISME), Genova, Italy
}

\begin{abstract}
The paper proposes a general framework to manage a team of Autonomous Underwater Vehicles (AUVs), while keeping the communication constraints, during missions execution. Virtual spring-damper couplings (passive by definition) define the distributed interaction forces between neighbouring vehicles. In this way, through passivity theory, a suitable Lyapunov function for the closed loop system is built to ensure stable convergence of the network vehicles to an equilibrium point, also providing robustness in presence of communication fading and delays, very common in the marine environment. Simulations of typical missions show the effectiveness of the proposed approach. An equivalence between this typical port-Hamiltonian framework and a specific class of potential games, the Bilateral Simmetric Interaction (BSI) one, is also established. Hence, modelling the network with passive elements, it is possible to shape the transient behaviour of the players and the reached equilibria at the end of the game.
\end{abstract}

Keywords: autonomous vehicles, cooperation, distributed control, passivity, port-Hamiltonian, potential games.

\section{Introduction}

A renewal of interest has been focused recently on the analysis and control of networked systems, and particularly on distributed systems of mobile agents. Such systems provide significant benefits in efficiency, scalability, and robustness when compared to classical centralized solutions. Applications of mobile agent networks are multi-disciplinary and highly diversified. In particular, recent advances in marine robotics have made AUVs more reliable and affordable allowing the execution of tasks that are dangerous, expensive and time consuming when performed by humans. There are a lot of practical applications that can benefit from the use of a team of AUVs: these include the defence field, patrolling, surveillance of an asset or of a predefined geographical area, coverage tasks, exploration, oceanographic sur-

\footnotetext{
This work was partially funded by European Union, programme H2020, Project WiMUST, grant n. 645141, and by DLTM, Ligurian Cluster on Marine Technology, Project RIMA.

${ }^{*}$ Corresponding author Fabiani)

Email address: filippo.fabiani@ing.unipi.it (Filippo
}

Preprint submitted to Ocean Engineering veying and mapping [1]. All these application scenarios involve communication among multiple agents. In the underwater domain, due to the well known limitations of the acoustic channel [2, 3, 4], it is of paramount importance to maintain desired communication performances in order to achieve the mission objectives.

Basing on recent preliminary works developed by the authors [5, 6], this paper presents a general framework for coordinating a team of agents, applied to a group of AUVs. The passivity theory is exploited for guaranteeing the stable and robust convergence of the network to an equilibrium configuration, even in presence of communication delays. The term "stability" here is used to indicate that, for any initial condition which ensures the fulfilment of the communication constraints, such constraints are satisfied for the whole transient, and the proposed algorithm leads the vehicles to assume a stable, in classic Lyapunov sense, configuration. Moreover, the degrees of freedom offered by such an approach allow to tune the desired motion of the group in terms of transient behaviour and reached equilibria. In particular, it is possible to determine in advance which task has higher priority than the others without any conse- 
quence about stability. Furthermore, the behaviour of the group is made more flexible, with arbitrary split and join events, using an "energy tank" able to store and supply energy whenever required [7]: exploiting this further passive element the network topology may change depending on the emerging needs of the mission.

Downstream to what just said, an equally relevant motivation behind this work arises from the possibility to capture an ambitious and innovative point of contact between two different ways to model and control a network of agents: the one proposed here and BSI, a potential game that exhibits symmetries across the variables. In fact, a BSI game is characterized by the notion of pairwise or bilateral strategic interaction, in which the utility function for each player depends only on its own action and that of another connected with him, whatever the behaviour of the rest of the network. If that happens for all players, the observations are said to be symmetric for each combination of connected agents. Hence, modelling the network with passive elements, which are purely design parameters, it may be possible to shape the transient behaviour of the players and, ideally, the reached equilibrium at the end of the game.

The proposed approach is based on the positive influences of several pioneering works, such as the one in [8], refined and extended in [9] and [10]. Here the artificial potentials and virtual leaders allowed to manage a group of multiple autonomous vehicles, also manipulating the team geometry and its direction of motion. Motivated by biological inspiration, such works focused also on gradient climbing missions in which the mobile sensor network sought out local maxima or minima in the environmental field; moreover, a convergent cooperative Kalman filter for exploration missions provided the estimates to drive the centre of the formation to move along level curves of the environmental field. In [11], natural potential functions were obtained from structural constraints of a desired formation: in this way, the synthesized controller for each vehicle was able to steer and move agents exploiting only local informations, also avoiding collisions. With the same concepts in mind, the author of [12] proposed a theoretical framework for the design and analysis of several distributed flocking algorithms, in presence or lack of obstacles. Another pioneering work that exploited artificial potentials to solve the constrained coverage problem is in [13]. In particular, the deployment of the mobile sensor network was addressed: each node was treated as a virtual charged particle, in order to synthesize an algorithm able to maximize the covered area and minimize the number of nodes of the network itself. Recently, in $[14,15]$, a mobility control that switch between a set of smooth, constraint-enforcing potential fields, satisfying local and non-local constraints composition was proposed. That potential-based control also drove the agents to maximize connectivity and maintain established links; the constraint satisfaction was achieved using a switched model of interaction which regulated link addition through repulsive potentials between constraint violators.

In the recent years, the authors proposed cooperative control algorithms based on the behavioural approach paradigm [16] and its adaptation as potential BSI game [17], to maintain desired communication performance and fulfil each agent task. The main drawback of such algorithms were the absence of stability guarantees along the whole motion of the agents: as a matter of fact, they were able to provide only the local stability of equilibria points. In this context, the port-Hamiltonian framework allows to model the sensors network in a suitable, passive fashion, e.g. in $[18,19,20]$. Passivity techniques have been widely studied in the domain of bilateral teleoperations for the control of a traditional single-master/single-slave system [21, 22], or for a more complex single-master/multiple-slaves system [23]. In spite of take advantage of the operator's intelligence for solving complex tasks as in bilateral teleoperations, the proposed framework seeks to provide full autonomy to the agents in order to accomplish the cooperative mission.

The paper is organized as follows: Section 2 presents the essentials mathematical and theoretical tools implied in the framework, i.e. graph theory, portHamiltonian systems and game theory; Section 3 outlines the implementation details of the cooperative algorithm and demonstrates the stability of the proposed solution including delays on communication links. After that, Section 4 provides several consideration about the relationship between the proposed approach and BSI games. Section 5 exploits the energy tanks approach proposed in [23] to enlarge the set of possible stable manoeuvres within the network, while Section 6 illustrates and discusses the effectiveness of the proposed framework in several application scenarios. Finally, Section 7 summarizes the work and draws the main conclusions.

Notation. $\mathbb{R}, \mathbb{R}_{>0}$ and $\mathbb{R}_{\geq 0}$ respectively denote the set of real, positive real, non-negative real numbers. Vectors and matrices are denoted by bold characters, while scalars and sets by italics. Unless otherwise specified, $\mathbf{0}$ and $\mathbf{I}$ denotes the zero and identity matrices of suitable dimensions (context-dependent). $\mathbf{A}^{\top}$ denotes the transpose of $\mathbf{A} ; \mathbb{S}^{n}$ denotes the set of symmetric $n \times n$ matrices; for a given $\mathbf{Q} \in \mathbb{R}^{n \times n}$, the notations $\mathbf{Q}>0(\mathbf{Q} \succcurlyeq 0)$ 
and $\mathbf{Q} \in \mathbb{S}_{\succ 0}^{n}\left(\mathbf{Q} \in \mathbb{S}_{\succcurlyeq 0}^{n}\right)$ denote that $\mathbf{Q}$ is symmetric and has positive (non-negative) eigenvalues. $|\cdot|$ denotes the cardinality of a set, $\|\cdot\|$ the Euclidean norm, $\otimes$ and $\times$ the Kronecker and Cartesian product, respectively.

\section{Background and Preliminaries}

This section provides the essentials from the main analytical and theoretical tools required for the upcoming mathematical treatment. In particular, some reminders on graph theory, passivity, port-Hamiltonian systems and game theory will be very useful for a proper understanding. We remark here that we are using the jargon of different, though converging, research fields. Therefore, in the rest of the paper we may refer to the AUVs with the terms agents, vehicles or players, indistinctly.

\subsection{Graph Theory}

A graph $\mathcal{G}:=(V, E)$ is formally defined by a finite set of nodes (or vertices) $V$ and a set of edges $E \subset V \times V$, connecting pairs of nodes. The node set $V:=\left\{v_{1}, v_{2}, \ldots, v_{l}\right\}$ has $l=|V|$ elements, while the edge set $E:=\left\{e_{1}, e_{2}, \ldots, e_{m}\right\}$ contains $m=|E|$ elements. Given $e_{j} \in E$, then there exist a pair $v_{i}, v_{j} \in V$ such that $e_{j}:=\left(v_{i}, v_{j}\right)$; in this way, $v_{i}$ and $v_{j}$ are said to be adjacent, while $\left(v_{i}, v_{i}\right)$ is called a self-loop. If the edges in graphs are to be interpreted as enabling information to flow between the vertices on the corresponding edge, these flows can be directed as well as undirected. Hence, direct and indirect graph can be distinguished. In the first case, edges have a fixed direction (i.e. the tail and the head of the edge are setted), while in the second case, if $\left(v_{i}, v_{j}\right)$ belongs to $E$, then $\left(v_{j}, v_{i}\right)$ belongs to $E$ too. However, for indirect graph, one can arbitrarily assign an orientation to each edge. Any key feature of a graph can be described by means of matrices. In particular, the incidence matrix $B(\mathcal{G})$ is a $l \times m$ matrix defined as follows:

$$
[B(\mathcal{G})]_{i j}:=b_{i j}= \begin{cases}-1 & \text { if } v_{i} \text { is the tail of } e_{j}, \\ 1 & \text { if } v_{i} \text { is the head of } e_{j}, \\ 0 & \text { otherwise. }\end{cases}
$$

The $l$ rows of $B(G)$ correspond to the nodes of $\mathcal{G}$, while the $m$ columns denotes the edges of such graph. For further details on the graph theory, refer to [24].

\subsection{Port-Hamiltonian Systems and Passivity}

The port-Hamiltonian framework, introduced in [25], allows to model complex (non-linear) systems as energy storing and energy dissipating components, connected via ports to power conserving transmissions and conversions. It is an energy-based framework in which each element interacts with the system via a port, that consists of a couple of dual effort and flow quantities, whose product gives the power flow in and out of the component. As well described in [26], let $\mathbf{x} \in \mathbb{R}^{n}$ denotes the local coordinates for an $n$-dimensional state space manifold $\mathcal{X}, \mathbf{u} \in \mathbb{R}^{m}$ the control input and $\mathbf{y} \in \mathbb{R}^{m}$ the output of the system. The generalized input-state-output dynamics expressed in terms of port-Hamiltonian framework is given by:

$$
\left\{\begin{array}{l}
\dot{\mathbf{x}}=[\mathbf{J}(\mathbf{x})-\mathbf{R}(\mathbf{x})]\left(\frac{\partial H(\mathbf{x})}{\partial \mathbf{x}}\right)^{\top}+\mathbf{g}(\mathbf{x}) \mathbf{u}, \\
\mathbf{y}=\mathbf{g}^{\top}(\mathbf{x})\left(\frac{\partial H(\mathbf{x})}{\partial \mathbf{x}}\right)^{\top}
\end{array}\right.
$$

where $\mathbf{J}(\cdot) \in \mathbb{R}^{n \times n}$ is a skew-symmetric structure matrix, $\mathbf{g}(\cdot) \in \mathbb{R}^{n \times m}$ is also a structure matrix, $H: \mathbb{R}^{n} \rightarrow \mathbb{R}_{\geq 0}$ is the Hamiltonian function that represents the whole energy stored in the system and $\mathbf{R}(\cdot) \in \mathbb{S}_{\succcurlyeq 0}^{n}$ a dissipation matrix. The entries of both matrices $\mathbf{J}(\cdot)$ and $\mathbf{R}(\cdot)$ depend smoothly on $\mathbf{x}$. Modelling dynamical system as (1) provides several benefits: in particular, a basic property of a port-Hamiltonian system is related to its energy balance, tightly coupled with the notion of passivity. In fact, any port-Hamiltonian system is passive w.r.t. the supply rate and storage function $H(\cdot)$ if $H(\cdot)$ is bounded from below.

Let now consider a generic, affine, non-linear system

$$
\left\{\begin{array}{l}
\dot{\mathbf{x}}=\mathbf{f}(\mathbf{x})+\mathbf{g}(\mathbf{x}) \mathbf{u} \\
\mathbf{y}=\mathbf{h}(\mathbf{x})
\end{array}\right.
$$

with the state vector $\mathbf{x} \in \mathbb{R}^{n}$, a control vector $\mathbf{u} \in \mathbb{R}^{m}$ and an output vector $\mathbf{y} \in \mathbb{R}^{m}$. f : $\mathbb{R}^{n} \rightarrow \mathbb{R}^{n}, \mathbf{g}: \mathbb{R}^{n} \rightarrow \mathbb{R}^{m}$ and $\mathbf{h}: \mathbb{R}^{n} \rightarrow \mathbb{R}^{m}$ are non-linear functions of the state.

Definition 1. (from [27])

The system in (2) is passive if there exists a continuous and differentiable lower bounded function of the state $V: \mathbb{R}^{n} \rightarrow \mathbb{R}_{\geq 0}$ (called storage function) such that:

$\dot{V}(\mathbf{x}) \leq \mathbf{u}^{\top} \mathbf{y} \Longleftrightarrow V(\mathbf{x}(t))-V(\mathbf{x}(0)) \leq \int_{0}^{t} \mathbf{u}^{\top}(\tau) \mathbf{y}(\tau) d \tau$.

$V(\cdot)$ represents the internal stored energy and $\mathbf{u}^{\top} \mathbf{y}$ the energy flow exchanged with the external world (i.e. the supply rate). The pair $(\mathbf{u}, \mathbf{y})$ is called power port, where $\mathbf{u}$ and $\mathbf{y}$ are power variables: these latter allow to control and interconnect passive systems.

Back to the port-Hamiltonian theory, due to the fact that $H(\mathbf{x}) \geq 0$, the passivity is always guaranteed and 
it is easy to show that $\dot{H}(\mathbf{x}) \leq \mathbf{u}^{\top} \mathbf{y}$. In this way, portHamiltonian framework provides a powerful tool for the stability analysis of dynamical systems, in order to achieve a feasible, stable and robust control.

\subsection{Game Theory and Nash Equilibria}

A game $\mathcal{G}:=\left[\mathcal{N}, \mathbb{A},\left\{U_{i}\right\}_{i \in \mathcal{N}}\right]$ in strategic, noncooperative form is defined by a non-empty and finite set $\mathcal{N}$ of $N$ rational players, a strategy space $\mathbb{A}$ and a set $\left\{U_{i}\right\}_{i \in \mathcal{N}}$ of utility functions. A player is rational if he or she makes decisions which are consistent to the purpose of maximizing (minimizing) his/her utility function. Mathematically speaking, let us denote by $\mathcal{N}:=\{1,2, \ldots, N\}$ the set of $N$ participating players. For every $i$-th player in $\mathcal{N}$, his/her collection of all possible strategies forms a strategy set, expressed as:

$$
\mathbf{A}_{i}:=\left\{A_{i} \mid A_{i} \text { is a valid strategy for the } i \text {-th player }\right\} .
$$

In this way, the strategy space $\mathbb{A}$ is defined as the Cartesian products of all individual strategy sets, i.e.:

$$
\mathbb{A}:=\mathbf{A}_{1} \times \ldots \times \mathbf{A}_{N} .
$$

Each element $A=\left(A_{1}, A_{2}, \ldots, A_{N}\right) \in \mathbb{A}$ is said to be a strategy profile. Referring to the $i$-th player, then $A$ can be rewritten as $A:=\left(A_{i}, A_{-i}\right)$, where $A_{-i}$ denotes the joint strategy adopted by player $i$ 's opponents. Hence, the domain of $A_{-i}$ is defined by $\mathbf{A}_{-i}$. For each player $i$, his/her utility function $U_{i}$ is a function that maps each strategy profile $A$ to a real number, that is $U_{i}: \mathbb{A} \rightarrow \mathbb{R}$. Consequently, $U_{i}(A)$ can be represented also as $U_{i}\left(A_{i}, A_{-i}\right)$.

A Nash equilibrium (NE) is a crucial concept in predicting game outcome: it is a strategy profile such that if the opponents' strategies remain unaltered, no player would be tempted to move away from his/her current strategy. More precisely:

Definition 2. The strategy profile $A^{*} \in \mathbb{A}$ is a $N E$ if and only if

$$
\forall A_{i}^{\prime} \in \mathbf{A}_{i}, \forall i \in \mathcal{N}, U_{i}\left(A_{i}^{*}, A_{-i}^{*}\right) \geq U_{i}\left(A_{i}^{\prime}, A_{-i}^{*}\right) .
$$

At a NE, no player is able to gain by deviating from the current point: it is a sort of "stable operating point". Although a NE is often the ultimate objective of a game, such game in strategic/normal form may have either unique, multiple or no NE.

\subsubsection{Potential Games}

As stated in [28] a pure strategy, normal form, potential game has the strong property that admits at lest one NE. By definition:

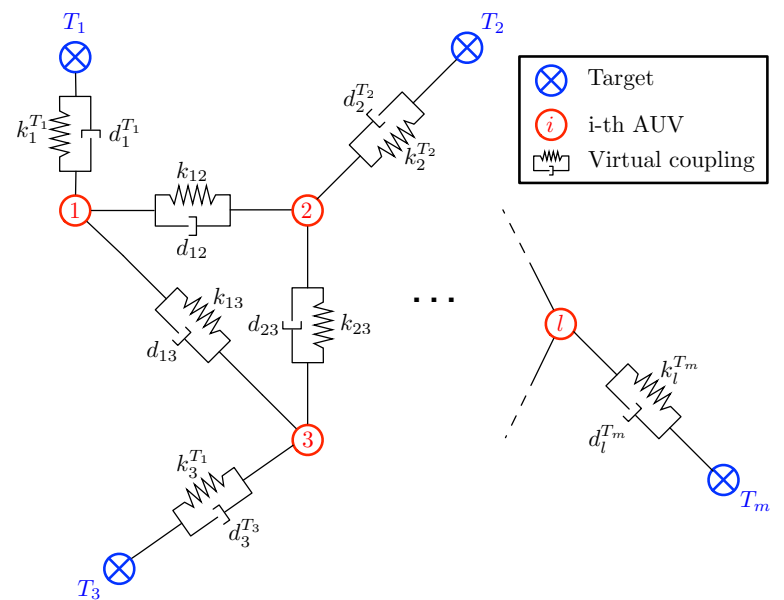

Figure 1: A team of $l$-AUVs (numbered red circles) in a generic distribution in $\mathbb{R}^{n}$. The blue circled crosses suggest the target of the agents, while the spring-damper couples represent the virtual couplings between agents and/or targets.

Definition 3. A game $\mathcal{G}$ is an exact potential game if and only if a potential function $V: \mathbb{A} \rightarrow \mathbb{R}$ exists such that, $\forall i \in \mathcal{N}$ :

$$
\begin{aligned}
U_{i}\left(T_{i}, A_{-i}\right)-U_{i}\left(A_{i}, A_{-i}\right)= & V\left(T_{i}, A_{-i}\right)-V\left(A_{i}, A_{-i}\right), \\
& \forall A_{i}, T_{i} \in \mathbf{A}_{i}, \forall A_{-i} \in \mathbf{A}_{-i} .
\end{aligned}
$$

In an exact potential game, the change in a single player's utility due to his/her own strategy deviation results in exactly the same amount of change in the potential function. One interesting property of such games is that the global maximizers (minimizers) of the potential function $V$ are NE.

\section{The High-Level Cooperative Algorithm}

In this paper, the general issue addressed is the following:

Problem. Each AUV within a team has to accomplish its own task (or tasks), while keeping the communication connectivity with the other team members.

\subsection{Networked System Model}

Modelling the agents/targets network as an indirect graph without any self-loop, the aim is to design a highlevel control policy that allows to maintain the predefined graph during the development of the whole mission.

From now on, we suppose that the following mild assumptions hold: 
Assumption 1. The connectivity link is guaranteed if the agents lie within a fixed relative range.

Assumption 2. Each vehicle has to know its own absolute velocity and the relative position of another vehicle with respect to itself.

It is worth nothing that in order to fulfil the maintenance of the communication link it is assumed that the performance of the acoustic channel, potential packet loss issues or Signal-to-Noise Ratio (SNR) have been converted in a range constraint. This is always possible (i.e., underwater acoustic communication difficulties decreases as communication ranges get shorter), although sometime too conservative. However, how to exploit the channel characteristics to optimize communication ranges [2] is out of the scope of the paper. The information required in Assumption 2 is measured or estimated by the available navigation system of the vehicles (see [29] for a recent review). Depending on the specific situation, or the specific set of vehicles, some sensors or some approaches can be more appropriate than another: for instance, in shallow water or for sea-bed inspection missions the presence of Doppler Velocity Log (DVL) sensor on board each vehicle is sufficient to measure the velocity. Acoustic modems with Ultra-Short Base Line (USBL) capability can not only deliver measurements of relative position, but can also concur to the establishment of a cooperative navigation system [30] which exploits range and bearing information as communication among the vehicles take place. Additional refinements for deep water and to compensate current disturbance can be found in [31]. A complete treatment of the navigation issue is clearly out of the scope of this paper; here it is assumed that a navigation system is in place, and that the required information is available to the vehicles.

Let now consider the generic network configuration in Fig. 1, with $l$-vehicles and $m$-targets. Each link, either a communication connection or not, is represented by a pair of spring-damper element (virtual coupling, passive by definition). The graph $\mathcal{G}:=(V, E)$ associated to the concerned network is defined by:

$$
\begin{aligned}
& V:=\left\{1,2, \ldots, l, T_{1}, \ldots, T_{m}\right\} \rightarrow|V|=l+m, \\
& E:=\left\{(1,2), \ldots,\left(1, T_{1}\right), \ldots\right\} \rightarrow|E|=a+t .
\end{aligned}
$$

Note the separation between agent-agent and agenttarget edges, with cardinality $a$ and $t$, respectively. Considering the $(i, j)$ edge, here the vertex $i$ is always seen as the tail and $j$ as the head of the link. Then, the inci- dence matrix $\mathbf{B} \in \mathbb{R}^{(l+m) \times(a+t)}$ is given by:

$$
\mathbf{B}=\left[\begin{array}{ccc:cc}
-1 & -1 & \cdots & -1 & \cdots \\
1 & 0 & \cdots & 0 & \ldots \\
0 & 1 & \cdots & 0 & \ldots \\
\vdots & \vdots & & \vdots & \\
\hdashline 0 & 0 & \cdots & 1 & \ldots \\
0 & 0 & \ldots & 0 & \ldots \\
\vdots & \vdots & & \vdots & \ldots \\
0 & 0 & \ldots & 0 & \ldots
\end{array}\right]
$$

Conventionally, the rows of $\mathbf{B}$ are sorted as the elements in $V$, while the columns as those in $E$. In this way, the first $a$ columns represent the links between agents and the second $t$ stand for the agent-target connections. For sake of clarity, assuming a simple damped double-integrator dynamics in $n$-dimensional space for each node of the network, the $i$-th AUV and $j$-th target equations of motion are given by:

$$
m_{i} \ddot{\mathbf{q}}_{\mathbf{i}}=-d \dot{\mathbf{q}}_{\mathbf{i}}+\mathbf{u}_{\mathbf{i}}, \quad m_{T_{j}} \ddot{\mathbf{q}}_{\mathbf{T}_{\mathbf{j}}}=-d \dot{\mathbf{q}}_{\mathbf{T}_{\mathbf{j}}}+\mathbf{u}_{\mathbf{T}_{\mathbf{j}}},
$$

where $m_{i}, m_{T_{j}}$ are the respective masses, $\mathbf{q}_{\mathbf{i}}, \mathbf{q}_{\mathbf{T}_{\mathbf{j}}} \in$ $\mathbb{R}^{n}$ are their positions (generalized coordinates) and $\mathbf{u}_{\mathbf{i}}, \mathbf{u}_{\mathbf{T}_{\mathbf{j}}} \in \mathbb{R}^{n}$ are the control vectors. The $d$ element model a viscous friction acting on the moving nodes. Hence, the corresponding linear momenta $\mathbf{p}_{\mathbf{i}}, \mathbf{p}_{\mathbf{T}_{\mathbf{j}}} \in \mathbb{R}^{n}$ are:

$$
\mathbf{p}_{\mathbf{i}}=\overline{\mathbf{m}}_{\mathbf{i}} \dot{\mathbf{q}}_{\mathbf{i}}, \quad \mathbf{p}_{\mathbf{T}_{\mathbf{j}}}=\overline{\mathbf{m}}_{\mathbf{T}_{\mathbf{j}}} \dot{\mathbf{q}}_{\mathbf{T}_{\mathrm{j}}},
$$

with $\overline{\mathbf{m}}_{\mathbf{i}}:=\mathbf{I} m_{i}, \overline{\mathbf{m}}_{\mathbf{T}_{\mathbf{j}}}:=\mathbf{I} m_{T_{j}}$ and $\mathbf{I}$ is the $n \times n$ identity matrix. Now, consider the group of $l+m$ vertices: to compactly denote the agent dynamics, the vector form is introduced. Thus, $\mathbf{q}=\left[\mathbf{q}_{1}{ }^{\top}, \mathbf{q}_{\mathbf{2}}{ }^{\top}, \ldots, \mathbf{q}_{\mathbf{T}_{\mathbf{1}}}{ }^{\top}, \ldots\right]^{\top} \in$ $\mathbb{R}^{n(l+m)}$ stands for the generalized coordinates vector, $\mathbf{D}^{\mathbf{A}}:=\operatorname{diag}(\mathbf{I} d, \ldots, \mathbf{I} d) \in \mathbb{R}^{n(l+m) \times n(l+m)}$ is the damping matrix and $\mathbf{u}=\left[\mathbf{u}_{\mathbf{1}}{ }^{\top}, \mathbf{u}_{\mathbf{2}}{ }^{\top}, \ldots, \mathbf{u}_{\mathbf{T}_{\mathbf{1}}}{ }^{\top}, \ldots\right]^{\top} \in \mathbb{R}^{n(l+m)}$ is the control vector. The linear momenta vector $\mathbf{p} \in$ $\mathbb{R}^{n(l+m)}$ is:

$$
\mathbf{p}=\mathbf{M} \dot{\mathbf{q}},
$$

where $\mathbf{M}:=\operatorname{diag}\left(\overline{\mathbf{m}}_{\mathbf{1}}, \ldots, \overline{\mathbf{m}}_{\mathbf{T}_{\mathbf{m}}}\right) \in \mathbb{S}_{\succ 0}^{n(l+m) \times n(l+m)}$ is the matrix of masses. Basing on the communication links, the relative distances vector $\mathbf{z} \in \mathbb{R}^{n(a+t)}$ is defined as:

$$
\mathbf{z}:=\left(\mathbf{B}^{\top} \otimes \mathbf{I}\right) \mathbf{q} .
$$

\subsection{Energetic Description of the Network}

Including the virtual couplings, the energy behaviour of the network is now analysed. The global Hamiltonian function $H: \mathbb{R}^{n(l+m)} \times \mathbb{R}^{n(a+t)} \rightarrow \mathbb{R}_{\geq 0}$ :

$$
H(\mathbf{p}, \mathbf{z})=H^{k}(\mathbf{p})+H^{t}(\mathbf{z})+H^{a}(\mathbf{z}),
$$


is divided as the sum of three different terms, where the potential contribution of the springs is split between AUV-target and AUV-AUV contributions. In details:

a) Kinematic contribution $H^{k}: \mathbb{R}^{n(l+m)} \rightarrow \mathbb{R}_{\geq 0}$

$$
H^{k}(\mathbf{p}):=\frac{1}{2} \mathbf{p}^{\top} \mathbf{M}^{-1} \mathbf{p} .
$$

b) Agent-Target contribution $H^{t}: \mathbb{R}^{n t} \rightarrow \mathbb{R}_{\geq 0}$

$$
H^{t}(\mathbf{z}):=\frac{1}{2} \mathbf{z}^{\top} \mathbf{K}_{\mathbf{T}} \mathbf{z},
$$

with $\mathbf{K}_{\mathbf{T}}:=\operatorname{diag}\left(\mathbf{0}, \overline{\mathbf{K}}_{\mathbf{T}}\right) \in \mathbb{R}^{n(a+t) \times n(a+t)}, \overline{\mathbf{K}}_{\mathbf{T}}:=$ $\operatorname{diag}\left(\overline{\mathbf{k}}_{\mathbf{1}}, \ldots, \overline{\mathbf{k}}_{\mathbf{t}}\right), \overline{\mathbf{k}}_{\mathbf{i}}:=\mathbf{I} k_{i}^{t}$ and $k_{i}^{t}$ is the elastic constant of the $i$-th vehicle-target coupling in $E$ (for example, $k_{1}^{t}=k_{1}^{T_{1}}$ and $k_{t}^{t}=k_{l}^{T_{m}}$ in Fig. 1, for $i=1, \ldots, t)$.

c) Agent-Agent contribution $H^{a}: \mathbb{R}^{n a} \rightarrow \mathbb{R}_{\geq 0}$

Defining $R_{c}$ as the maximum distance at which the AUV can still communicate with its neighbours, and $R_{d} \leq R_{c}$ as the desired distance to be maintained between two vehicles, the last term of $H(\mathbf{p}, \mathbf{z})$ is given by:

$$
H^{a}(\mathbf{z}):=\frac{1}{2} \sum_{i=1}^{a} k_{i}^{a}\left(\left\|\mathbf{z}_{\mathbf{i}}\right\|\right) \cdot\left(\left\|\mathbf{z}_{\mathbf{i}}\right\|-R_{d}\right)^{2},
$$

where the elastic constant $k_{i}^{a}(\cdot)$ models a non-linear spring over each agent-agent link.

Defining $\mathbf{f} \in \mathbb{R}^{n(a+t)}$ as the force vector acting on the $a+t$ links:

$$
\mathbf{f}=\left(\frac{\partial H}{\partial \mathbf{z}}\right)^{\top}+\mathbf{D}^{\mathbf{C}}\left(\mathbf{B}^{\top} \otimes \mathbf{I}\right)\left(\frac{\partial H}{\partial \mathbf{p}}\right)^{\top},
$$

where $\mathbf{D}^{\mathbf{C}}:=\operatorname{diag}\left(\overline{\mathbf{d}}_{\mathbf{1}}, \ldots, \overline{\mathbf{d}}_{\mathbf{a}}, \ldots, \overline{\mathbf{d}}_{\mathbf{t}}\right) \in \mathbb{S}_{>0}^{n(a+t) \times n(a+t)}$ contains the mutual damping elements $\overline{\mathbf{d}}_{\mathbf{i}}:=\mathbf{I} d_{i}$ in $E$. In this way, assuming $(\mathbf{p}, \mathbf{z})$ as the state variables, the input-state representation of the multi-agents open loop system in the port-Hamiltonian framework is:

$$
\begin{aligned}
& {\left[\begin{array}{c}
\dot{\mathbf{p}} \\
\dot{\mathbf{z}}
\end{array}\right]=\left[\begin{array}{c:c}
-\mathbf{D}^{\mathbf{A}} & \mathbf{0} \\
\hdashline \mathbf{B}^{\top} \otimes \mathbf{I} & \mathbf{0}
\end{array}\right]\left[\begin{array}{l}
\left(\frac{\partial H}{\partial \mathbf{p}}\right)^{\top} \\
\left(\frac{\partial H}{\partial \mathbf{z}}\right)^{\top}
\end{array}\right]+\left[\begin{array}{c}
\mathbf{I} \\
\mathbf{0}
\end{array}\right] \mathbf{u}} \\
& \mathbf{y}=\left[\begin{array}{l:l}
\mathbf{I} & \mathbf{0}
\end{array}\right]\left[\begin{array}{c}
\left(\frac{\partial H}{\partial \mathbf{p}}\right)^{\top} \\
\left(\frac{\partial H}{\partial \mathbf{z}}\right)^{\top}
\end{array}\right] .
\end{aligned}
$$

Now, Pfaffian constraints are introduced to generalize the proposed framework and then to consider fixed nodes (e.g. the targets). In this way, after choosing a suitable matrix $\mathbf{A} \in \mathbb{R}^{n(l+m) \times n m}$ that satisfies the relation $\mathbf{A}^{\top}\left(\frac{\partial H}{\partial \mathbf{p}}\right)^{\top}=\mathbf{0}$, a sort of selection matrix $\mathbf{S} \in$
$\mathbb{R}^{n(l+m) \times n(l+m)}$ such that $\mathbf{A}^{\top} \mathbf{S}=\mathbf{0}$ can be easily computed. Then, the open loop system in (4) assumes the following form:

$$
\begin{aligned}
& {\left[\begin{array}{c}
\dot{\mathbf{p}} \\
\dot{\mathbf{z}}
\end{array}\right]=\left[\begin{array}{c:c}
-\mathbf{S}^{\top} \mathbf{D}^{\mathbf{A}} \mathbf{S} & \mathbf{0} \\
\hdashline\left(\mathbf{B}^{\top} \otimes \mathbf{I}\right) \mathbf{S} & \mathbf{0}
\end{array}\right]\left[\begin{array}{c}
\left(\frac{\partial H}{\partial \mathbf{p}}\right)^{\top} \\
\left(\frac{\partial H}{\partial \mathbf{z}}\right)^{\top}
\end{array}\right]+\left[\begin{array}{c}
\mathbf{S} \\
\hdashline \mathbf{0}
\end{array}\right] \mathbf{u}} \\
& \mathbf{y}=\mathbf{S}^{\top}\left(\frac{\partial H}{\partial \mathbf{p}}\right)^{\top} .
\end{aligned}
$$

\subsection{Control Synthesis and Stability}

In order to synthesize a control law that stabilizes the system and allows to meet the communication constraints, the Hamiltonian function $H(\mathbf{p}, \mathbf{z})$ is now chosen as the Lyapunov candidate for the multi-agents multitargets network. Indeed, differentiating with respect to time:

$$
\dot{H}(\mathbf{p}, \mathbf{z})=\frac{\partial H^{k}}{\partial \mathbf{p}} \dot{\mathbf{p}}+\frac{\partial}{\partial \mathbf{z}}\left(H^{t}+H^{a}\right) \dot{\mathbf{z}},
$$

and replacing the dynamic (5) in (6), hence:

$$
\begin{aligned}
\dot{H}(\mathbf{p}, \mathbf{z}) & =\frac{\partial H}{\partial \mathbf{p}} \mathbf{S u}-\frac{\partial H}{\partial \mathbf{p}} \mathbf{S}^{\top} \mathbf{D}^{\mathbf{A}} \mathbf{S}\left(\frac{\partial H}{\partial \mathbf{p}}\right)^{\top}+ \\
& +\frac{\partial H}{\partial \mathbf{p}} \mathbf{S}^{\top}\left(\mathbf{B}^{\top} \otimes \mathbf{I}\right)\left(\frac{\partial H}{\partial \mathbf{z}}\right)^{\top} .
\end{aligned}
$$

Defining $\overline{\mathbf{S}}:=\left(\mathbf{B}^{\top} \otimes \mathbf{I}\right) \mathbf{S}$ and choosing the control vector u as:

$$
\mathbf{S u}=-\overline{\mathbf{S}}^{\top}\left(\frac{\partial H}{\partial \mathbf{z}}\right)^{\top}-\overline{\mathbf{S}}^{\top} \mathbf{D}^{\mathbf{C}} \overline{\mathbf{S}}\left(\frac{\partial H}{\partial \mathbf{p}}\right)^{\top},
$$

the autonomous closed loop dynamics becomes:

$$
\left[\begin{array}{c}
\dot{\mathbf{p}} \\
\dot{\mathbf{z}}
\end{array}\right]=\left[\begin{array}{c:c}
-\left(\mathbf{S}^{\top} \mathbf{D}^{\mathbf{A}} \mathbf{S}+\overline{\mathbf{S}}^{\top} \mathbf{D}^{\mathbf{C}} \overline{\mathbf{S}}\right) & -\overline{\mathbf{S}}^{\top} \\
\hdashline \overline{\mathbf{S}} & \mathbf{0}
\end{array}\right]\left[\begin{array}{l}
\left(\frac{\partial H}{\partial \mathbf{p}}\right)^{\top} \\
\left(\frac{\partial H}{\partial \mathbf{z}}\right)^{\top}
\end{array}\right] .
$$

Proposition 1. The proposed control law in (7) leads the trajectories of the closed loop system (8) within the set given by $(\partial H / \partial \mathbf{z})^{\top} \in \operatorname{ker}\left\{\overline{\mathbf{S}}^{\top}\right\}$, i.e. at the equilibrium point between the elastic forces generated to the agentagent, agent-target links.

Proof. Just invoking the La Salle principle, applied to the obtained negative semi-definite $\dot{H}(\mathbf{p}, \mathbf{z})$, the trajectories of (8) converge to the largest invariant set where $\mathbf{p}=\mathbf{0}$, that is:

$$
-\overline{\mathbf{S}}^{\top}\left(\frac{\partial H}{\partial \mathbf{z}}\right)^{\top}=\mathbf{0} .
$$




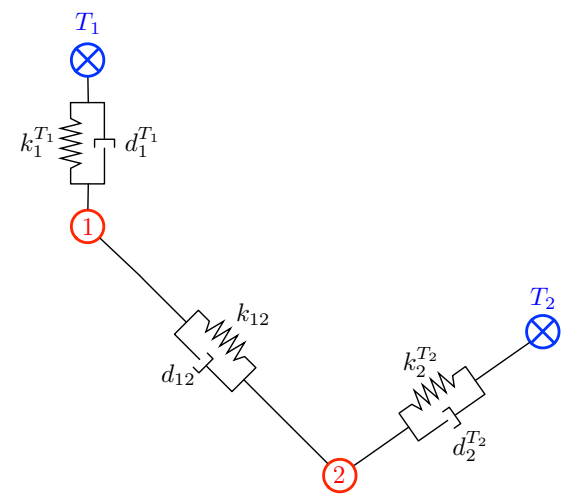

Figure 2: A generic distribution in $\mathbb{R}^{2}$ for the two AUVs - two targets case.

Note that the control law in (7) is distributed. In fact, each AUV can compute its control input knowing only the relative position and velocity of the agents connected with itself. Recalling the Assumption 2, the only information which requires to be shared within the team is hence the absolute velocity of each vehicle in the horizontal plane. Such an information can conveniently be coded in a data packet containing two floats and sent with a broadcast message by each vehicle on the communication channel. Note that such communication load, in addition to being small ( 8 bytes), it is also scalable w.r.t. the number of connected agents.

Moreover, as regards the choice of the control gains, the proposed control law offers several degrees of freedom, as well as strong stability guarantees. In fact, selecting different elastic constants, it is possible to determine in advance which task has higher priority than the other without any consequence about the stability property.

Example 1. The discussion just presented is now applied to the trivial case of two agents - two targets acting in $\mathbb{R}^{2}$ (Fig. 2). The aim is to provide a clearer interpretation of the invariant set in (9) that contains the trajectories of the controlled system. So, the set $E=\left\{(1,2),\left(1, T_{1}\right),\left(2, T_{2}\right)\right\}$ contains the different connectivity links, the selection matrix $\mathbf{S}=\operatorname{diag}\left(\mathbf{I}^{4 \times 4}, \mathbf{0}^{4 \times 4}\right)$ and the incidence matrix is:

$$
\mathbf{B}=\left[\begin{array}{c:cc}
-1 & -1 & 0 \\
1 & 0 & -1 \\
\hdashline 0 & 1 & 0 \\
0 & 0 & 1
\end{array}\right]
$$

Choosing a linear spring to model the agent-agent virtual coupling (i.e. $k_{1}^{a}\left(\left\|\mathbf{z}_{1}\right\|\right)=k_{1}^{a}$ ), the system (8) is stabilized to the set of $\mathbf{z}$ that fulfil the following system of equations:

$$
\left\{\begin{array}{r}
k_{1}^{a}\left(1-\frac{R_{d}}{\left\|\mathbf{z}_{\mathbf{1 2}}\right\|}\right) z_{12_{x}}+k_{1}^{t} z_{1 T_{1 x}}=0 \\
k_{1}^{a}\left(1-\frac{R_{d}}{\left\|\mathbf{z}_{\mathbf{1 2}}\right\|}\right) z_{12_{y}}+k_{1}^{t} z_{1 T_{1 y}}=0 \\
-k_{1}^{a}\left(1-\frac{R_{d}}{\left\|\mathbf{z}_{\mathbf{1 2}}\right\|}\right) z_{12_{x}}+k_{2}^{t} z_{2 T_{2 x}}=0 \\
-k_{1}^{a}\left(1-\frac{R_{d}}{\left\|\mathbf{z}_{12}\right\|}\right) z_{12_{y}}+k_{2}^{t} z_{2 T_{2 y}}=0
\end{array}\right.
$$

where

$\mathbf{z}_{\mathbf{1 2}}=\left[\begin{array}{c}z_{12_{x}} \\ z_{12_{y}}\end{array}\right], \quad \mathbf{z}_{\mathbf{1} \mathbf{T}_{\mathbf{1}}}=\left[\begin{array}{c}z_{1 T_{1 x}} \\ z_{1 T_{1 y}}\end{array}\right], \quad \mathbf{z}_{2 \mathbf{T}_{\mathbf{2}}}=\left[\begin{array}{c}z_{2 T_{2 x}} \\ z_{2 T_{2 y}}\end{array}\right]$,

represent the relative distances ordered in $E$, while $k_{1}^{a}$, $k_{1}^{t}$ and $k_{2}^{t}$ the respective elastic constants.

\subsection{Stability to Communication Delays}

The required information to be exchanged among neighbouring vehicles (velocity) could be affected by communication delays on the connectivity links. This is particularly critical in marine acoustic communications, due to the speed of sound in the ocean (typical value $1500 \mathrm{~m} / \mathrm{s}$ ). Before stating the main result of this section, we make the following assumption, which will be clear later:

Assumption 3. The time varying delay acting on each connectivity link is represented by $\tau_{i}(t)$, a non-negative, bounded above, Lipschitz function.

Thus, we are ready to state the following theorem, which is a generalization of the result of [32]:

Theorem 1. Under Assumption 3, if there exist ( $a+$ t) matrices $\overline{\mathbf{P}}_{\mathbf{i}} \in \mathbb{S}_{>0}^{n(l+m) \times n(l+m)}$ that meet the following Linear Matrix Inequalities (LMI) problem:

$$
-\mathbf{S}^{\top} \mathbf{D}^{\mathbf{A}} \mathbf{S}-\overline{\mathbf{D}}+\sum_{i=1}^{a+t} \overline{\mathbf{P}}_{\mathbf{i}} \prec \mathbf{0},
$$

then Proposition 1 still holds even in presence of communication delays.

Proof. The control policy synthesized above can be restated as:

$$
\mathbf{S u}=-\overline{\mathbf{S}}^{\top}\left(\frac{\partial H}{\partial \mathbf{z}}\right)^{\top}-\left[\overline{\mathbf{D}}\left(\frac{\partial H}{\partial \mathbf{p}}\right)^{\top}-\sum_{i=1}^{a+t} \overline{\mathbf{T}}_{\mathbf{i}}\left(\frac{\partial H}{\partial \mathbf{p}}\right)_{\tau_{i}(t)}^{\top}\right],
$$

where: 
- $\overline{\mathbf{D}}$ contains the diagonal elements of $\overline{\mathbf{S}}^{\top} \mathbf{D}^{\mathrm{C}} \overline{\mathbf{S}}$;

- $\overline{\mathbf{T}}_{\mathbf{i}}$ contains the out of diagonal elements of $\overline{\mathbf{S}}^{\top} \mathbf{D}^{\mathrm{C}} \overline{\mathbf{S}}$ closely related with $\tau_{i}(t)$.

The term $(\partial H / \partial \mathbf{p})_{\tau_{i}(t)}$ represents the partial derivative of the Hamiltonian function w.r.t. the linear momenta, computed in according to the time varying delay on the $i$-th link. Omitting the time dependence of each $\tau_{i}$ and defining:

$$
\nabla H^{\top}:=\left[\begin{array}{c}
\left(\frac{\partial H}{\partial \mathbf{p}}\right)^{\top} \\
\left(\frac{\partial H}{\partial \mathbf{z}}\right)^{\top}
\end{array}\right], \quad \nabla H_{\tau_{i}}^{\top}:=\left[\begin{array}{c}
\left(\frac{\partial H}{\partial \mathbf{p}}\right)_{\tau_{i}}^{\top} \\
\left(\frac{\partial H}{\partial \mathbf{z}}\right)_{\tau_{i}}^{\top}
\end{array}\right],
$$

the Lyapunov candidate $V: \mathbb{R}^{n(l+m)} \times \mathbb{R}^{n(a+t)} \rightarrow \mathbb{R}_{\geq 0}$ is now chosen as in [32]:

$$
V(\mathbf{p}, \mathbf{z})=H(\mathbf{p}, \mathbf{z})+\sum_{i=1}^{a+t} \int_{t-\tau_{i}}^{t} \nabla H_{s_{i}} \mathbf{P}_{\mathbf{i}} \nabla H_{s_{i}}^{\top} d s_{i},
$$

with each $\mathbf{P}_{\mathbf{i}} \in \mathbb{S}_{\succcurlyeq 0}^{n(l+m+a+t) \times n(l+m+a+t)}$. In view of the Assumption 3 the time derivative function of (10) is hence:

$$
\begin{aligned}
\dot{V}(\mathbf{p}, \mathbf{z})=\dot{H}(\mathbf{p}, \mathbf{z})+ & \sum_{i=1}^{a+t}\left[\nabla H \mathbf{P}_{\mathbf{i}} \nabla H^{\top}+\right. \\
& \left.-\left(1-\dot{\tau}_{i}\right) \nabla H_{\tau_{i}} \mathbf{P}_{\mathbf{i}} \nabla H_{\tau_{i}}^{\top}\right] .
\end{aligned}
$$

Noting that only the term $(\partial H / \partial \mathbf{p})_{\tau_{i}}$ of $\nabla H_{\tau_{i}}$ is really affected by lag, hence $\mathbf{P}_{\mathbf{i}}=\operatorname{diag}\left(\overline{\mathbf{P}}_{\mathbf{i}}, \mathbf{0}\right)$. Then, considering the worst case in which each $\dot{\tau}_{i}=\tilde{d}$ and compacting everything in vector form, after some simple algebraic manipulations:

$$
\dot{V}(\mathbf{p}, \mathbf{z}) \leq\left[\begin{array}{c}
\left(\frac{\partial H}{\partial \mathbf{p}}\right)^{\top} \\
\left(\frac{\partial H}{\partial \mathbf{p}}\right)_{\tau}^{\top}
\end{array}\right]^{\top} \mathbf{W}\left[\begin{array}{c}
\left(\frac{\partial H}{\partial \mathbf{p}}\right)^{\top} \\
\left(\frac{\partial H}{\partial \mathbf{p}}\right)_{\tau}^{\top}
\end{array}\right],
$$

where:

$$
\left(\frac{\partial H}{\partial \mathbf{p}}\right)_{\tau}^{\top}:=\left[\begin{array}{c}
\left(\frac{\partial H}{\partial \mathbf{p}}\right)_{\tau_{1}}^{\top} \\
\vdots \\
\left(\frac{\partial H}{\partial \mathbf{p}}\right)_{\tau_{a+t}}^{\top}
\end{array}\right],
$$

and the matrix $\mathbf{W}$ is:

$\left[\begin{array}{c:ccc}-\mathbf{S}^{\top} \mathbf{D}^{\mathbf{A}} \mathbf{S}-\overline{\mathbf{D}}+\sum_{i=1}^{a+t} \overline{\mathbf{P}}_{\mathbf{i}} & \overline{\mathbf{T}}_{\mathbf{1}} & \ldots & \overline{\mathbf{T}}_{\mathbf{a}+\mathbf{t}} \\ \hdashline \mathbf{0} & -(1-\tilde{d}) \overline{\mathbf{P}}_{\mathbf{1}} & \ldots & \mathbf{0} \\ \vdots & \vdots & \ddots & \vdots \\ \mathbf{0} & \mathbf{0} & \ldots & -(1-\tilde{d}) \overline{\mathbf{P}}_{\mathbf{a}+\mathbf{t}}\end{array}\right]$

In this way, the time derivative of the Lyapunov candidate in (11) is negative definite iff $\mathbf{W} \prec \mathbf{0}$. Due to the fact that $\mathbf{W}$ is a diagonal matrix and that $-(1-\tilde{d}) \overline{\mathbf{P}}_{\mathbf{i}}<\mathbf{0}$ is satisfied for all possible choices of $\overline{\mathbf{P}}_{\mathbf{i}}$, the only condition that should be verified is the one on the first entry and this conclude the proof.

Remark 1. Assuming a positive, bounded above, Lipschitz delay function $\tau_{i}(t)$, as stated in Assumption 3, is quite realistic in marine environment. As a matter of fact, there are two main reasons why the time derivative of the delay is greater than one: if $c$ is the speed of sound in water, the first trivial case implies that two vehicles are moving at a relative velocity that is greater than $c$ (quite uncommon). The second reason is closely related with the packet loss, shown in the following example.

Example 2. Consider two fixed nodes $(\mathcal{A}, \mathcal{B})$, at a predefined distance $R_{0}$ : the node $\mathcal{A}$ transmits messages, while $\mathcal{B}$ receives them. Trivially, the time derivative of the delay $\tau$ can be approximated by:

$$
\dot{\tau} \simeq \frac{\Delta \tau}{\Delta t}
$$

If $\mathcal{A}$ sends at time $t_{0}$, then $\mathcal{B}$ successfully receives the message at time $t_{0}+\tau$. If a second message, sent at time $t_{1}$, it is not properly received at $t_{1}+\tau$, the node $\mathcal{A}$ ideally re-sends such message at $t_{1}+\tau$. In the unlikely event that it is not properly received again, the node $\mathcal{A}$ sends the same message for the third time at $t_{1}+2 \tau$ (as many commercial acoustic modems work), that is correctly received by $\mathcal{B}$ at $t_{1}+3 \tau$. Thus, considering this unfortunate set of transmissions, the final delay is equal to $3 \tau$, so $\Delta \tau=3 \tau-\tau=2 \tau$, while $\Delta t \geq 3 \tau$. Then, the following chain of inequalities holds:

$$
\dot{\tau} \simeq \frac{\Delta \tau}{\Delta t} \leq \frac{2}{3}<1
$$

The example above show that, even in the ideal case of immediate retransmissions of the lost packet, assuming $|\dot{\tau}|<1$ is still reasonable. Such assumption, for instance, was always verified in the field tests reported in [30].

\section{Relationship with Potential BSI Games}

This section aims to introduce the BSI games and to show the close relation with the proposed way of model a network of interconnected agents. 


\subsection{Symmetric Observations and BSI Games}

BSI games are characterized by bilateral or pairwise strategic interactions. For the $i$-th player, the utility function $U_{i}$ contains a term $w_{i j}\left(A_{i}, A_{j}\right)$, namely the observation seen by $i$ due to the strategy of $j$, which takes place solely due to the pairwise interaction between him/her and another player $j$. A BSI game is a game where the observations are symmetric, i.e. $w_{i j}\left(A_{i}, A_{j}\right)=$ $w_{j i}\left(A_{j}, A_{i}\right)$, across all pairs of players [33]. That is, $\forall i, j \in \mathcal{N}, i \neq j$, there exist functions $w_{i j}: \mathbf{A}_{i} \times \mathbf{A}_{j} \rightarrow \mathbb{R}$ such that $w_{i j}\left(A_{i}, A_{j}\right)=w_{j i}\left(A_{j}, A_{i}\right)$ for all $A_{i} \in \mathbf{A}_{i}$ and $A_{j} \in \mathbf{A}_{j}$. Moreover, the utility function of player $i$ is assumed to be of the form:

$$
U_{i}\left(A_{i}, A_{-i}\right)=\sum_{j \neq i} w_{i j}\left(A_{i}, A_{j}\right), \forall i \in \mathcal{N} .
$$

As reported in [33], the next theorem shows that BSI games are exact potential games (the proof is here omitted for sake of brevity).

Theorem 2. Assume $G$ is a BSI game. Then it is also an exact potential game with the following potential function:

$$
V(A)=\sum_{i \in \mathcal{N}} \sum_{\substack{j \in \mathcal{N}, j<i}} w_{i j}\left(A_{i}, A_{j}\right)
$$

Note that the original utility function proposed in [33] is obtained combining BSI games and no-conflict games. Due to the linear combination property of utility functions [34], the resulting exact potential game has utility functions of the form $U_{i}(A)=\sum_{j \neq i} w_{i j}-P_{i}\left(A_{i}\right)$, where $P_{i}: A_{i} \rightarrow \mathbb{R}$ indicates the no-conflict term. Hence, the potential function can be rewritten as:

$$
V(A)=\sum_{i \in \mathcal{N}} \sum_{\substack{j \in \mathcal{N}, j<i}} w_{i j}\left(A_{i}, A_{j}\right)-\sum_{i \in \mathcal{N}} P_{i}\left(A_{i}\right)
$$

\subsection{Equivalence Relations}

With the previous concepts in mind, if the strategy set for the $i$-th agent in Fig. 1 is $A_{i}:=\left(\mathbf{p}_{i}, \mathbf{q}_{i}\right)$, consider its general Hamiltonian function:

$$
h_{i}\left(A_{i}, A_{-i}\right):=\underbrace{\frac{1}{2} \sum_{k \in \mathbb{N}_{i}^{a}} h^{a_{k}}\left(\mathbf{z}_{\mathbf{k}}\right)+\frac{1}{2} \sum_{j \in \mathbb{N}_{i}^{t}} h^{t_{j}}\left(\mathbf{z}_{\mathbf{j}}\right)}_{\sum_{q \in \mathbb{N}_{i}} w_{i q}\left(\mathbf{z}_{q}\right)}+\underbrace{\frac{1}{2} \mathbf{p}_{i}^{\top} \overline{\mathbf{m}}_{i}^{-1} \mathbf{p}_{i}}_{-P_{i}\left(\mathbf{p}_{i}\right)},
$$

where $\mathbb{N}_{i}^{a}$ and $\mathbb{N}_{i}^{t}$ are the sets of agents and targets connected with the $i$-th agent, while $\mathbb{N}_{i}$ contains all that links, i.e. $\mathbb{N}_{i}=\mathbb{N}_{i}^{a} \cup \mathbb{N}_{i}^{t}$. Note that the strategies of the neighbouring agents $A_{-i}$ are included in $\left\{\mathbf{z}_{q}\right\}_{q \in \mathbb{N}_{i}}$, coupled with $A_{i}$. In this way, the functions $h^{a_{k}}(\cdot)$ and $h^{t_{j}}(\cdot)$, i.e. the springs contributions (possibly non-linear), depend only on the relative distance between two nodes; it is straightforward to observe that the action of the spring on the nodes is the same: from here, the symmetry of the observations in the proposed framework. The kinematic contribution instead $\mathbf{p}_{i}^{\top} \overline{\mathbf{m}}_{i}^{-1} \mathbf{p}_{i}$ represents a sort of pure coordination term, because no agent connected with the $i$-th cares about which action is taken from it.

Now, assuming the $i$-th Hamiltonian function as the $i$-th player's utility function, it is quite easy to show that the global Hamiltonian in (3) can be rewritten as the potential function of the game in (12), just imposing without abuse $w_{i j}=0$ on non-existing links. In fact:

$$
\begin{aligned}
& -\sum_{i \in \mathcal{N}} P_{i}\left(A_{i}\right)=\frac{1}{2} \sum_{i=1}^{l+m} \mathbf{p}_{i}^{\top} \overline{\mathbf{m}}_{i}^{-1} \mathbf{p}_{i}=H^{k}(\mathbf{p}), \\
& \sum_{i \in \mathcal{N}} \sum_{\substack{j \in \mathcal{N}, j<i}} w_{i j}\left(A_{i}, A_{j}\right)=\sum_{i \in \mathcal{N}} \sum_{q \in \mathbb{N}_{i}} w_{i q}\left(\mathbf{z}_{q}\right)=H^{t}(\mathbf{z})+H^{a}(\mathbf{z}) .
\end{aligned}
$$

Remark 2. If there are some fixed nodes, the potential contribution of the springs depends only on the strategy of the considered agent. It also becomes a pure coordination term.

Remark 3. The Hamiltonian function in (3) is a convex function in the generalized coordinates $(\mathbf{p}, \mathbf{z})$. The resolution of the minimization problem, that leads to a $N E$ of the game, is not trivial due to the presence of the communication constraints and fixed nodes: these may induce several local minima in the potential function and make richer the invariant set (9). However, in the simple case where there are no constraints (i.e. any spring with equilibrium point different from 0) or fixed nodes, the set of strategies that minimizes the Hamiltonian function is the one that leads the agent in the NE $(\mathbf{0 ,}, \mathbf{0})$.

In the authors' previous works (and here), as in [16] and [17], the desired intra-agents behaviour was obtained by means of non-linear springs, whose elastic potential was given by:

$$
h^{a_{i}}\left(\mathbf{z}_{\mathbf{i}}\right):= \begin{cases}K_{1}\left(\left\|\mathbf{z}_{\mathbf{i}}\right\|-R_{d}\right)^{2} & \left\|\mathbf{z}_{\mathbf{i}}\right\| \leq R_{d} \\ K_{2} \frac{\left(\left\|\mathbf{z}_{\mathbf{i}}\right\|-R_{d}\right)^{2}}{R_{c}-\left\|\mathbf{z}_{\mathbf{i}}\right\|} & \text { otherwise }\end{cases}
$$

where $K_{1}, K_{2}>0$ were design parameters. As an example, Fig. 3 shows the function in (13) with a typical value for the maximum communication range of a 


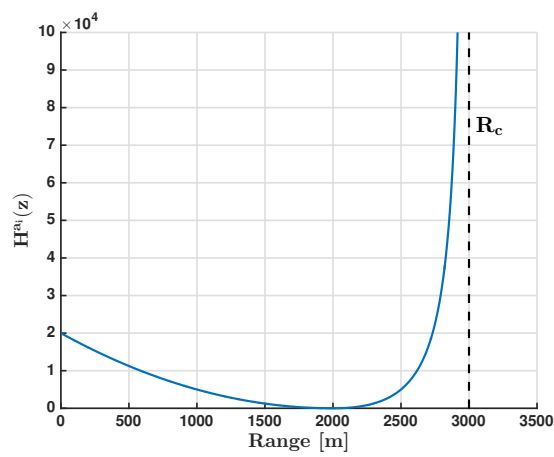

Figure 3: Elastic potential associated to an agent-agent connection, with $R_{c}=3000 \mathrm{~m}$ and $R_{d}=2000 \mathrm{~m}$.

medium frequency acoustic modem, e.g. $R_{c}=3000 \mathrm{~m}$, and $R_{d}=2000 \mathrm{~m}$.

Thus, the resulting gradient-based control rules were able to solve the general area coverage problem, reducing the overlap of sonar detection and ensuring that each agent maintains at least one other vehicle in its communication range. However, there were several limitations to the application of such control law: although the possibility to tune the appropriate interest functions (i.e. utility functions), the myopic coordination of AUVs could neither provide any guarantee of stability of the networked system, nor ability to predict which NE of the game was reached.

At a price of a slightly more complex network model (couples of spring-damper, incidence matrix, port-Hamiltonian framework and so on), with the control algorithm (7) presented in this work, that is also gradient-based and offers the same degrees of freedom of the previous one, it is always possible to arbitrarily change the transient behaviour of the network, maintaining the robust stability guarantees previously demonstrated. Moreover, exploiting the equivalence relations with potential games, it is possible to shape and determine in advance the potential functions, its set of local minima and the set of NE related with game.

\section{Integrating the energy tanks}

This section provides a modelling strategy for obtaining a flexible cohesive behaviour of the group of AUVs by applying the artificial tank approach introduced and demonstrated in [23]. As there stated, the passivity is guaranteed during a split manoeuvre, i.e. the cancellation of the coupling force between a pair of agents when they are outside the communication range. Not the same holds for a join manoeuvre, i.e. the re-establishment of

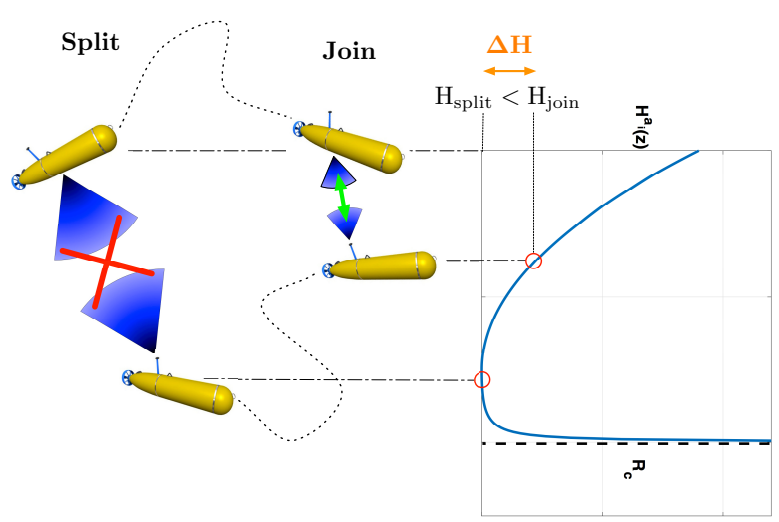

Figure 4: When the agents perform a join manoeuvre, it may occur that the required energy $H_{\text {join }}$ is greater than the one stored in the spring during the split manoeuvre $\left(H_{\text {split }}\right.$, without loss of generality in $0)$. In this way, the required extra amount of energy could breaks the passivity of the whole network. Note that the same holds if one of the two agents is replaced by a fixed target.

the coupling: as a matter of fact, some extra energy can still be produced during the join procedure. According to the scenario in Fig. 4, the relative distance between two agents at the join operation may induce to an higher elastic potential energy with respect to the one at the split operation, leading to an increase of the Hamiltonian function and consequently a loss of stability guarantees. This entails a new edge in the graph and the corresponding update of the incidence matrix B. However, the passivity of the global system can be maintained by endowing each AUV with a container of dissipated energy, called tank.

\subsection{Mathematical Model}

Due to the natural damping of the marine environment, the amount of power dissipated by each of $l$ mobile nodes is:

$$
D_{i}=\frac{\partial H}{\partial \mathbf{p}_{\mathbf{i}}} \overline{\mathbf{d}}^{\mathbf{A}}\left(\frac{\partial H}{\partial \mathbf{p}_{\mathbf{i}}}\right)^{\top},
$$

where $\overline{\mathbf{d}}^{\mathbf{A}}$ are the elements of the diagonal matrix $\mathbf{D}^{\mathbf{A}}$ (implicitly assuming that such natural damping is the same for all the AUVs). Thus, the dynamics of the $i$-th tank is:

$$
\dot{t}_{i}=\frac{1}{t_{i}} \theta_{i} D_{i}+\sum_{j \in \mathcal{N}_{i}} \mathbf{w}_{\mathbf{i j}}^{\top}\left(\frac{\partial H}{\partial \mathbf{z}_{\mathbf{i j}}}\right)^{\top},
$$

where $\mathcal{N}_{i}$ denotes the set of nodes connected with $i$, the parameter $\theta_{i} \in\{0,1\}$ disables/enables the storage of the energy dissipated by the vehicle and $\mathbf{w}_{\mathbf{i j}} \in \mathbb{R}^{n}$ is an input vector that allows to exchange energy between virtual springs and tanks. 
A few more words about $\theta_{i}$ and its usefulness must be spent. Although the introduced tanks are artificial, note that each of them has an associated energetic function $T_{i}=\frac{1}{2} t_{i}^{2}$. Filling the tanks with a huge amount of energy, indeed, could lead to some unstable behaviours in the whole system, as reported in [35]; in this way, it is conservative (but necessary) to disable the energy storage.

Thus, compacting in vector form and including the dynamics of tanks in (8), the augmented networked system with the proposed control law becomes:

$$
\left[\begin{array}{c}
\dot{\mathbf{p}} \\
\dot{\mathbf{z}} \\
\dot{\mathbf{t}}
\end{array}\right]=\left[\begin{array}{c:c:c}
-\left(\mathbf{S}^{\top} \mathbf{D}^{\mathbf{A}} \mathbf{S}+\overline{\mathbf{S}}^{\top} \mathbf{D}^{\mathbf{C}} \overline{\mathbf{S}}\right) & -\overline{\mathbf{S}}^{\top} & \mathbf{0} \\
\hdashline \overline{\mathbf{S}} & \mathbf{0} & \boldsymbol{\Gamma}^{\top} \\
\hdashline \boldsymbol{\Theta P} \mathbf{S}^{\top} \mathbf{D}^{\mathbf{A}} \mathbf{S} & -\boldsymbol{\Gamma} & \mathbf{0}
\end{array}\right]\left[\begin{array}{c}
\left(\frac{\partial \mathcal{H}}{\partial \mathbf{p}}\right)^{\top} \\
\left(\frac{\partial \mathcal{H}}{\partial \mathbf{z}}\right)^{\top} \\
\left(\frac{\partial \mathcal{H}}{\partial \mathbf{t}}\right)^{\top}
\end{array}\right],
$$

where $\mathcal{H}: \mathbb{R}^{n(l+m)} \times \mathbb{R}^{n(a+t)} \times \mathbb{R}^{a} \rightarrow \mathbb{R}_{\geq 0}$

$$
\mathcal{H}(\mathbf{p}, \mathbf{z}, \mathbf{t})=H(\mathbf{p}, \mathbf{z})+\frac{1}{2} \mathbf{t}^{\top} \mathbf{t},
$$

is the augmented total energy of the system, while (14) represents the autonomous behaviour of the whole network. In particular, $\boldsymbol{\Theta}:=\operatorname{diag}\left(\theta_{i}\right) \in \mathbb{R}^{(l+m)}, \mathbf{P}:=$ $\operatorname{diag}\left(\frac{1}{t_{i}} \mathbf{p}_{\mathbf{i}}^{\top} \overline{\mathbf{m}}_{\mathbf{i}}^{-T}\right) \in \mathbb{R}^{(l+m) \times n(l+m)}$ and the matrix $\boldsymbol{\Gamma} \in$ $\mathbb{R}^{(l+m) \times n(a+t)}$ is built as $\boldsymbol{\Gamma}_{i,(n(j-1)+1, \ldots, n j)}:=b_{i j} \mathbf{w}_{\mathbf{i j}}{ }^{\top}$. Although the time-varying nature of the incidence matrix $\mathbf{B}$, in [23] the authors show that, using $\mathcal{H}(\cdot)$ as a storage function, the system in (14) is passive w.r.t. external input acting on the agents. Thus, by appropriately choosing the matrix $\boldsymbol{\Gamma}$, i.e. the input vector $\mathbf{w}_{\mathbf{i j}}$, it is possible to prevent positive jumps in the storage function $\mathcal{H}(\cdot)$ during the join operations. Note that the time derivative of the energetic function associated to each tank is:

$$
\dot{T}_{i}=\dot{t}_{i} t_{i}=\theta_{i} D_{i}+t_{i} \sum_{j \in \mathbb{N}_{i}} \mathbf{w}_{\mathbf{i j}}^{\top}\left(\frac{\partial H}{\partial \mathbf{z}_{\mathbf{i j}}}\right)^{\top},
$$

where $\mathbb{N}_{i}$ is the set of neighbours of the agent $i$. In this way, choosing arbitrarily the sign of $\mathbf{w}_{\mathbf{i j}}$, it is possible to extract a certain amount of energy from $H^{a}(\cdot)$, injecting it into $T_{i}$ or vice versa.

Remark 4. The fact that split and join manoeuvring depends on the available energy stored in the artificial tank may rise the impression that only a limited amount of such manoeuvring is possible. Indeed, the artificial tank is filled by the motion of AUV itself, accumulating the energy dissipated during motion. This means that after a split-join manoeuvre, the vehicles have to wait a sufficient time to refill the tanks before attempting another split-join manoeuvre. In practice, this means that a too rapid sequence of split-join action (or too early in the mission, as at the very beginning) may disrupt network connectivity and ultimately stability. The dynamics of the artificial tanks sets implicitly a sufficiently large time interval among two consecutive splitjoin manoeuvres to guarantee passivity of the network.

As a final note, it is straightforward to demonstrate that the proposed modelling allows also to manage the dynamic reconfiguration of the network in case of fault of one or more vehicles without affecting the stability of the overall system. Indeed, a vehicle subject to a failure involuntarily behaves as a fixed node and the remainder of the team can continue the mission by executing a split manoeuvre with the faulty vehicle, without violating the passivity of the system. The maintenance of the stability in case of inclusion of additional agents in the team is more critical to be proven, as it involves a join manoeuvre among the new vehicle and the team. This, in principle, can be realised in a passive way as long as the energy stored in the tanks of the vehicles of the team is sufficient to compensate the energy introduced in the system by the new agent. However, this case is not considered in this work, since a formal demonstration must be treated carefully and will be subject of future investigations.

\subsection{The Stability Issue}

Here further aspects about the stability of the autonomous system in (14) and the invariant set reached by its trajectories are treated. In particular, the following proposition holds:

Proposition 2. The trajectories of the augmented system in (14) converge within the largest invariant set given by:

$$
\left\{\begin{array}{l}
\mathbf{0}=-\overline{\mathbf{S}}^{\top}\left(\frac{\partial \mathcal{H}}{\partial \mathbf{z}}\right)^{\top} \\
\dot{\mathbf{z}}=\boldsymbol{\Gamma}^{\top}\left(\frac{\partial \mathcal{H}}{\partial \mathbf{t}}\right)^{\top} \\
\dot{\mathbf{t}}=-\boldsymbol{\Gamma}\left(\frac{\partial \mathcal{H}}{\partial \mathbf{z}}\right)^{\top}
\end{array}\right.
$$

allowing "safe" join manoeuvres.

Proof. The networked system in (14) has an additional state $\mathbf{t}$ due to the introduced tanks dynamics. However, choosing the storage function in (15) as the new Lyapunov candidate and differentiating it w.r.t. time:

$$
\begin{aligned}
\dot{\mathcal{H}}(\mathbf{p}, \mathbf{z}, \mathbf{t})=\nabla \mathcal{H}\left[\begin{array}{c}
\dot{\mathbf{p}} \\
\dot{\mathbf{z}} \\
\dot{\mathbf{t}}
\end{array}\right] & =\frac{\partial \mathcal{H}}{\partial \mathbf{t}} \boldsymbol{\Theta P S}^{\top} \mathbf{D}^{\mathbf{A}} \mathbf{S}\left(\frac{\partial \mathcal{H}}{\partial \mathbf{p}}\right)^{\top}+ \\
& -\frac{\partial \mathcal{H}}{\partial \mathbf{p}}\left(\mathbf{S}^{\top} \mathbf{D}^{\mathbf{A}} \mathbf{S}+\overline{\mathbf{S}}^{\top} \mathbf{D}^{\mathbf{C}} \overline{\mathbf{S}}\right)\left(\frac{\partial \mathcal{H}}{\partial \mathbf{p}}\right)^{\top} .
\end{aligned}
$$


The second term in the RHS of (17) is always nonpositive (both $\mathbf{D}^{\mathbf{A}}$ and $\mathbf{D}^{\mathbf{C}}$ are positive definite matrices); then, making explicit the first one:

$$
\frac{\partial \mathcal{H}}{\partial \mathbf{t}} \boldsymbol{\Theta P} \mathbf{S}^{\top} \mathbf{D}^{\mathbf{A}} \mathbf{S}\left(\frac{\partial \mathcal{H}}{\partial \mathbf{p}}\right)^{\top}=\mathbf{t}^{\top} \boldsymbol{\Theta}\left[\begin{array}{c}
\frac{1}{t_{1}} D_{1} \\
\vdots \\
\frac{1}{t_{l}} D_{l}
\end{array}\right]=\sum_{i=1}^{l} \theta_{i} D_{i} .
$$

Noting that $\theta_{i} \in\{0,1\}$, such term represents at most the energy dissipated by the AUVs; hence, the second term is always greater than the first one, and $\dot{\mathcal{H}}(\mathbf{p}, \mathbf{z}, \mathbf{t}) \leq 0$. Again, invoking the La Salle principle applied to the obtained negative semi-definite $\dot{V}(\cdot)$, the trajectories of (14) converge to the largest invariant set where $\mathbf{p}=0$.

\section{Simulation results and discussion}

This section aims to show the simulation results obtained by applying the control algorithm presented in Section 3 to a team (or teams) of AUVs. Due to its flexibility, the proposed control law can be exploited to accomplish several cooperative missions, noting once again that the maintenance of the communication link among the vehicles belonging to a team (or a sub-team) is a fundamental requirement for the success of the collective task. Thus, after choosing a proper initial incidence matrix, the non-linear spring associated to each connection is modelled as reported in Section 4. In this way, defining an initial sub-graph between the agents of a team, the fulfilment of the communication constraints can be guaranteed: if two agents start within the communication range, they remain connected during the whole task, converging if possible to the desired distance $R_{d}$.

Remark 5. Generally, at the beginning of each mission, a support ship or a docking station allow the deployment of vehicles within a restricted area; so it is quite realistic to assume the existence of a communication link among vehicles from the beginning.

In the following, the simulation results obtained with several types of typical tasks are presented, where velocities are supposed to be exchanged between the vehicles at a rate of $1 \mathrm{~Hz}$. Moreover, all the simulations (except the last one in Section 6.3, which is delay-free) are performed considering a fixed targets and fixed communication delay of $1 \mathrm{~s}$.

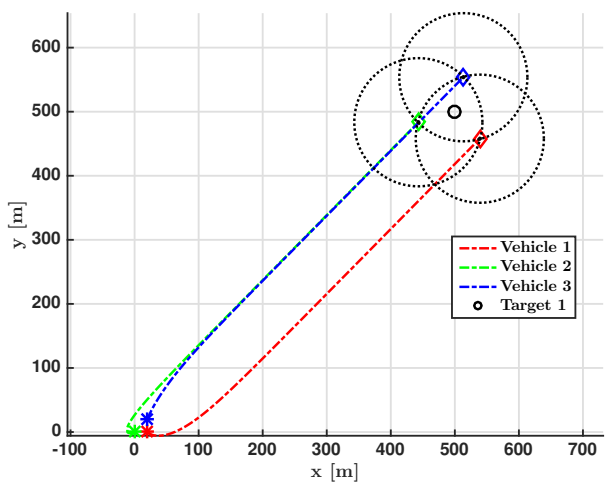

Figure 5: Cooperative surveillance task. The vehicles start from the positions indicated by the coloured stars. At the end, the vehicles (coloured diamonds) are disposed around the asset (black circle) while satisfying the distance constraints, represented with the dotted circles.
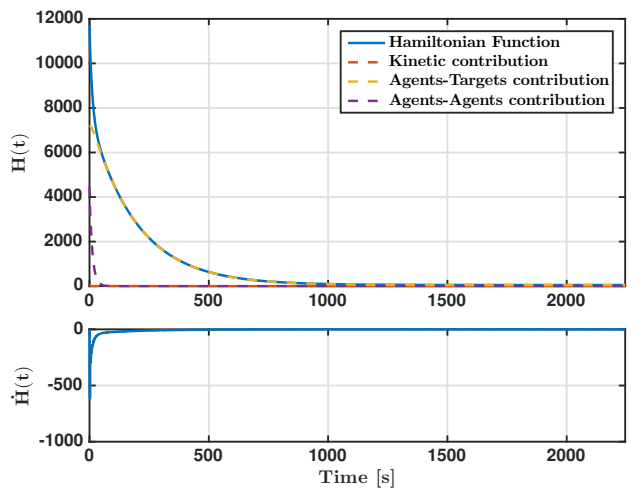

Figure 6: Hamiltonian function with each single contribution of (3) (top) and its time-derivative (bottom). 


\subsection{Patrolling: Single and Multiple Assets}

In a surveillance or patrolling task, the vehicles of a team have to cover a certain area around an asset to be defended. The first scenario in Fig. 5 involves only one target (the asset itself, $m=1$ ) and a team of three vehicles $(l=3)$. Hence, the agent-target connections are modelled by a spring-damper couple from each vehicle to the asset, setting instead the desired intra-agent range $R_{d}=100 \mathrm{~m}$. As it can be noticed, the team reaches a stable configuration around the asset to be defended. Furthermore, each vehicle remains within the desired distance with respect to all the others. In Fig. 6 the Hamiltonian function (3) and its time derivative are represented. Note that the function $\dot{H}(\cdot)$ is negative semidefinite, as expected with the choice of the control input defined in (7). In Fig. 7 there is another example of such task with two sub-teams of AUVs while performing a patrolling of multiple assets. Here there are two main assets and two sub-teams consisting of three and four vehicles, respectively (numbers 1-2-3 and 4-5-6-7); each target is connected by a virtual spring-damper couple with each agent of the corresponding sub-team. The communication constraints are defined among the vehicles belonging to the same sub-team. As it can be noticed in Fig. 8, each team reaches a stable configuration around the asset to be defended while the velocities decrease almost exponentially: this fact directly depends on the choice of the non-linear springs, which effects decrease as the AUVs get closer to the target. Due to the symmetry of the problem, the sub-team with three vehicles shapes an equilateral triangle around its target, while the one with four agents shapes a square. Moreover, each vehicle remains within the desired distance with respect to all others within the team (Fig. 9).

\subsection{Coverage Task}

In this case, each vehicle is assigned to a specific target $(l=m)$ with the overall objective of covering the maximum area in the targets' neighbourhood. Each coupling between an agent and the associated target is modelled by a spring-damper connection. Fig. 10 shows the trajectories followed by the team members in a scenario with three vehicles $(l=m=3)$ and a relative distance among the agents $R_{d}=2500 \mathrm{~m}$. In this case, the team reaches a stable configuration in which no vehicle can move closer to the corresponding target without breaking the imposed constraints due to the communication maintenance. As it can be seen from Fig. 11, after an initial transient in which it decreases, the Hamiltonian function settles at a constant value, different from zero. This is the effect of the agent-target springs that

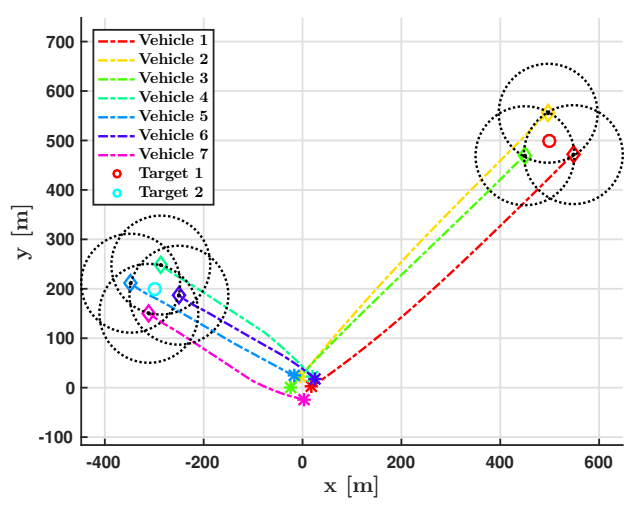

Figure 7: Cooperative patrolling of multiple assets. The two subteams start from the positions indicated by the coloured stars. At the end, the vehicles (coloured diamonds) are disposed around the assets (red and cyan circles) while satisfying the distance constraints, represented with the dotted circles.

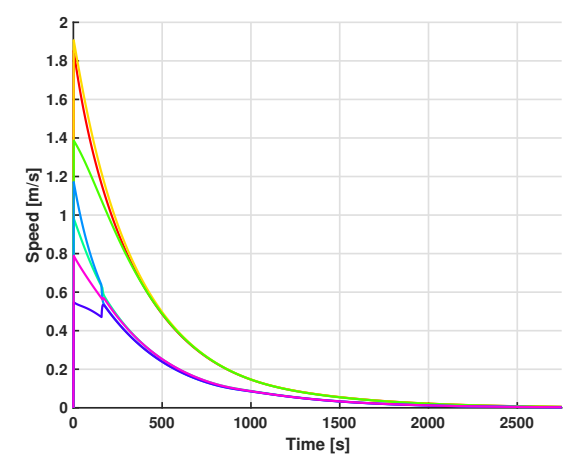

Figure 8: Velocities of the AUVs of the two sub-teams involved in the mission.

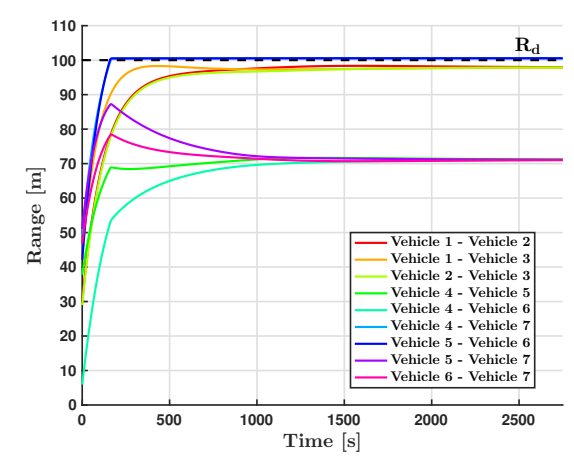

Figure 9: Relative distances among agents belonging to the same subteam. Note that the solid blue (5-6) and light blue (4-7) lines represent the virtual couplings between the agents placed on the two diagonal of the square: the communication constraint is still fulfilled. 


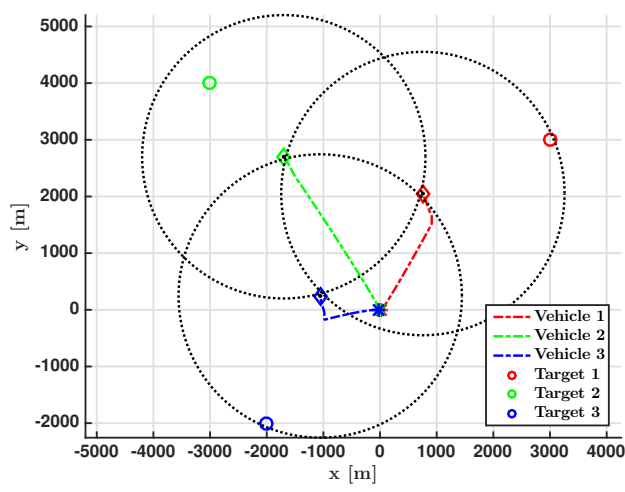

Figure 10: Cooperative coverage task. The vehicles start to approach to the assigned targets until the communication limit (dotted circles) is attained.
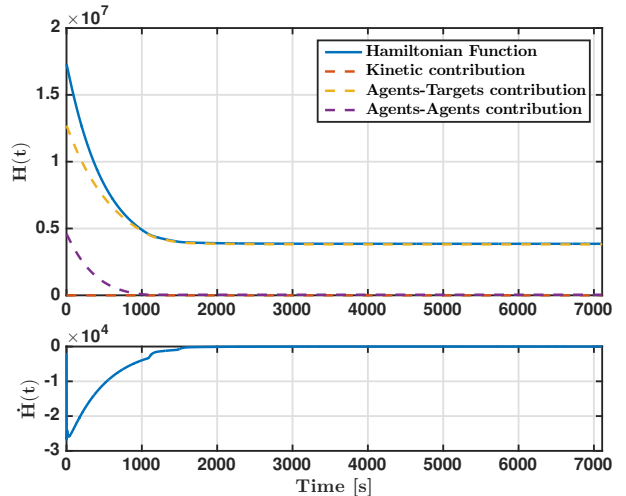

Figure 11: Hamiltonian function with the different contributions (top) and its time-derivative (bottom). do not reach their equilibrium point (the well-known invariant in (9)). As expected, the time-derivative of the Hamiltonian is still negative semi-definite.

\subsection{Exploration Task}

Given a team of AUVs and a set of significant spots, the fulfilment of an exploration task implies that each site is visited (i.e. reached within a certain tolerance $R_{T}$ ) at least once by a vehicle. Consider the case with three agents, that have to maintain the connectivity one to each other, and three possible targets. Depending on the sites location, even if the elastic constants on the agent-target connections are not the same, the proposed control law (7) leads the vehicles in a static, equilateral triangle shaped configuration (deadlock) that does not allow the accomplishment of the exploration task (e.g. as in the case of the coverage task in Fig. 10). In order to guarantee the success of the mission, one possible solution when a deadlock occur is to maintain only one agent-target link at time, breaking and regenerating the connections following a certain policy. In this case, the agents have assigned a predefined priority that schedule the spot exploration order: when a deadlock occurs, the agent with highest priority takes the "leadership" of the group, while the others "disconnect" themselves from their related targets (split manoeuvre) as long as the leader does not accomplish its sub-task. Now the story is trivial: the first leader breaks the connection with its target, a new leader is elected, e.g. the agent with immediately lower priority, and the corresponding connection is restored (join manoeuvre). Depending on the position reached by the new leader, this operation could require an injection of energy into the system (i.e. when the join manoeuvre happens at an higher distance from the target with respect to the split one). This amount of extra energy is picked up from the tank of the moving agent, implementing the algorithm described in Section 5. Note that the split and join manoeuvres can be executed also between agents, allowing to relax the assumption of the communication maintenance if possible or required. In this case, the required extra amount of energy would be extracted from both the tanks, accordingly with the procedure reported in [23].

\section{Conclusions and Future Developments}

Exploiting the port-Hamiltonian system modelling, jointly with the passivity theory, the proposed distributed control law allows to fulfil communication constraints between interconnected marine vehicles during the execution of several missions. Moreover, such 


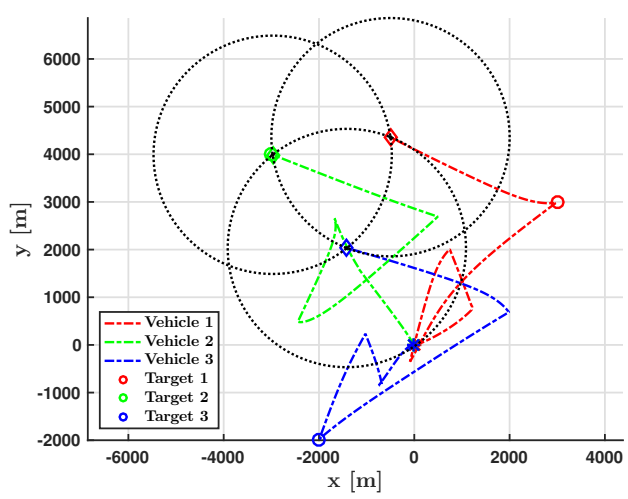

Figure 12: Exploration task. The agents start from the positions indicated by the coloured stars and reach the corresponding target (coloured circles) one at a time. In this case, the priority is 3-1-2.

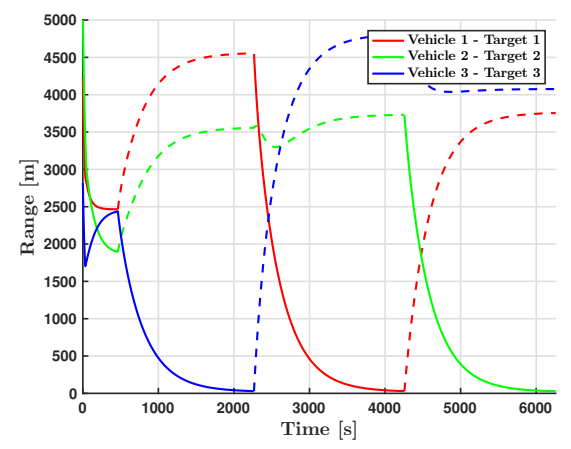

Figure 13: Relative distances between agents and corresponding target over time. The solid intervals of each line suggest the phase of link activation, while the dashed one represents the "absence" of connection.

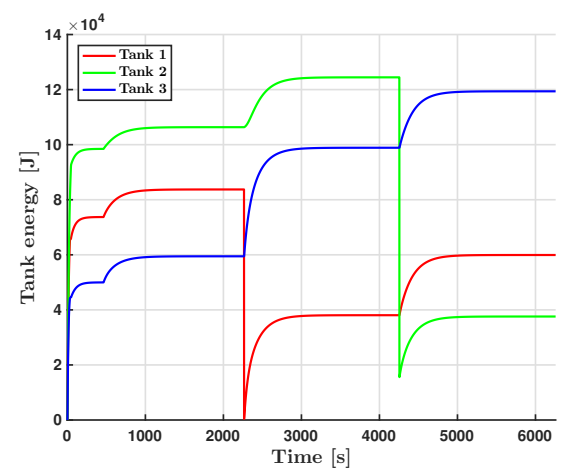

Figure 14: Tanks dynamics. During the evolution of the mission, the tank is charged up until it reaches a saturation. The downward spikes characterize the join manoeuvres, where the involved vehicle pick up an extra amount of energy from its tank. framework suggests an easy way to draw quick conclusions about the soundness and stability in the large of the whole system, providing robustness and stability guarantees even in presence of time varying delays on the connectivity links, which are very common in marine environment. The large number of degrees of freedom, that can be exploited upstream of the mission, represents one of the main features of the algorithm; as a matter of fact, each passive virtual coupling introduces an artificial potential that can be arbitrarily shaped, without any consequence about the stability of the sensors network. In this way, selecting different elastic constants, it is possible to determine in advance which task has higher priority, "choosing" the set of reachable equilibria. The behaviour of the group is also made flexible, with arbitrary split and joint events: the passivity property of the whole network is kept safe using artificial tanks, able to store and supply energy whenever required. With the setting of the present paper, the equivalence between the proposed framework and BSI games has been shown. Whether this equivalence can be formally extended to a larger class of systems it is an interesting challenge for future research.

\section{References}

[1] T. B. Curtin, J. G. Bellingham, J. Catipovic, D. Webb, Autonomous oceanographic sampling networks, Oceanography 6 (3) (1993) 86-94

[2] A. Caiti, K. Grythe, J. M. Hovem, S. M. Jesus, A. Lie, A. Munafò, T. A. Reinen, A. Silva, F. Zabel, Linking acoustic communications and network performance: integration and experimentation of an underwater acoustic network, IEEE Journal of Oceanic Engineering 38 (4) (2013) 758-771.

[3] M. Stojanovic, On the relationship between capacity and distance in an underwater acoustic communication channel, SIGMOBILE Mob. Comput. Commun. Rev. 11 (4) (2007) 34-43.

[4] A. Caiti, E. Crisostomi, A. Munafò, Physical characterization of acoustic communication channel properties in underwater mobile sensor networks., in: S-CUBE, Springer, 2009, pp. 111126.

[5] F. Fabiani, D. Fenucci, T. Fabbri, A. Caiti, A distributed, passivity-based control of autonomous mobile sensors in an underwater acoustic network, IFAC-PapersOnLine 49 (23) (2016) 367-372.

[6] F. Fabiani, D. Fenucci, T. Fabbri, A. Caiti, A passivity-based framework for coordinated distributed control of auv teams: Guaranteeing stability in presence of range communication constraints, in: OCEANS 2016 MTS/IEEE Monterey, IEEE, 2016, pp. $1-5$.

[7] C. Secchi, S. Stramigioli, C. Fantuzzi, Position drift compensation in port-hamiltonian based telemanipulation, in: 2006 IEEE/RSJ International Conference on Intelligent Robots and Systems, IEEE, 2006, pp. 4211-4216.

[8] N. E. Leonard, E. Fiorelli, Virtual leaders, artificial potentials and coordinated control of groups, in: Decision and Control, 2001. Proceedings of the 40th IEEE Conference on, Vol. 3, IEEE, 2001, pp. 2968-2973. 
[9] P. Ogren, E. Fiorelli, N. E. Leonard, Cooperative control of mobile sensor networks: Adaptive gradient climbing in a distributed environment, IEEE Transactions on Automatic control 49 (8) (2004) 1292-1302.

[10] F. Zhang, N. E. Leonard, Cooperative filters and control for cooperative exploration, IEEE Transactions on Automatic Control 55 (3) (2010) 650-663.

[11] R. Olfati-Saber, R. M. Murray, Distributed cooperative control of multiple vehicle formations using structural potential functions, in: IFAC World Congress, Vol. 15, Citeseer, 2002, pp. 242-248.

[12] R. Olfati-Saber, Flocking for multi-agent dynamic systems: Algorithms and theory, IEEE Transactions on automatic control 51 (3) (2006) 401-420.

[13] S. Poduri, G. S. Sukhatme, Constrained coverage for mobile sensor networks, in: Robotics and Automation, 2004. Proceedings. ICRA'04. 2004 IEEE International Conference on, Vol. 1, IEEE, 2004, pp. 165-171.

[14] R. K. Williams, G. S. Sukhatme, Locally constrained connectivity control in mobile robot networks, in: Robotics and Automation (ICRA), 2013 IEEE International Conference on, IEEE, 2013, pp. 901-906.

[15] R. K. Williams, G. S. Sukhatme, Topology-constrained flocking in locally interacting mobile networks, in: Robotics and Automation (ICRA), 2013 IEEE International Conference on, IEEE, 2013, pp. 2002-2007.

[16] A. Caiti, V. Calabrò, G. Dini, A. Lo Duca, A. Munafò, Secure cooperation of autonomous mobile sensors using an underwater acoustic network, Sensors 12 (2) (2012) 1967-1989.

[17] A. Caiti, T. Fabbri, D. Fenucci, A. Munafò, Potential games and AUVs cooperation: First results from the THESAURUS project, in: OCEANS - Bergen, 2013 MTS/IEEE, 2013, pp. 1-6.

[18] S. Fiaz, D. Zonetti, R. Ortega, J. Scherpen, A. Van der Schaft, A port-hamiltonian approach to power network modeling and analysis, European Journal of Control 19 (6) (2013) 477-485.

[19] E. Vos, J. M. Scherpen, A. J. van der Schaft, A. Postma, Formation control of wheeled robots in the port-hamiltonian framework, IFAC Proceedings Volumes 47 (3) (2014) 6662-6667.

[20] E. Vos, M. Jafarian, C. De Persis, J. M. Scherpen, A. J. Van Der Schaft, Formation control of nonholonomic wheeled robots in the presence of matched input disturbances, IFACPapersOnLine 48 (13) (2015) 63-68.

[21] P. F. Hokayem, M. W. Spong, Bilateral teleoperation: An historical survey, Automatica 42 (12) (2006) 2035-2057.

[22] C. Secchi, S. Stramigioli, C. Fantuzzi, Transparency in porthamiltonian-based telemanipulation, IEEE Transactions on Robotics 24 (4) (2008) 903-910.

[23] A. Franchi, C. Secchi, H. I. Son, H. H. Bulthoff, P. R. Giordano, Bilateral teleoperation of groups of mobile robots with time-varying topology, IEEE Transactions on Robotics 28 (5) (2012) 1019-1033.

[24] M. Mesbahi, M. Egerstedt, Graph theoretic methods in multiagent networks, Princeton University Press, 2010.

[25] B. Maschke, A. Van der Schaft, Port-controlled Hamiltonian systems: modelling origins and system theoretic properties, in: M. Fliess (Ed.), Nonlinear Control Systems Design 1992, IFAC Symposia Series, Pergamon, Oxford, 1993, pp. 359 - 365.

[26] A. Van der Schaft, L2-gain and passivity techniques in nonlinear control, Springer Science \& Business Media, 2006.

[27] V. Duindam, A. Macchelli, S. Stramigioli, H. Bruyninckx, Modeling and control of complex physical systems: the portHamiltonian approach, Springer Science \& Business Media, 2009.

[28] D. Monderer, L. S. Shapley, Potential games, Games and economic behavior 14 (1) (1996) 124-143.
[29] L. Paull, S. Saeedi, M. Seto, H. Li, AUV navigation and localization: A review, IEEE Journal of Oceanic Engineering 39 (1) (2014) 131-149.

[30] B. Allotta, A. Caiti, R. Costanzi, F. Di Corato, D. Fenucci, N. Monni, L. Pugi, A. Ridolfi, Cooperative navigation of AUVs via acoustic communication networking: field experience with the Typhoon vehicles, Autonomous Robots 40 (7) (2016) 12291244.

[31] B. Allotta, R. Costanzi, F. Fanelli, N. Monni, L. Paolucci, A. Ridolfi, Sea currents estimation during AUV navigation using Unscented Kalman Filter, IFAC-PapersOnLine 50 (1) (2017) 13668-13673.

[32] R. Pasumarthy, C.-Y. Kao, On stability of time delay hamiltonian systems, in: American Control Conference, 2009. ACC'09., IEEE, 2009, pp. 4909-4914.

[33] T. Ui, A shapley value representation of potential games, Games and Economic Behavior 31 (1) (2000) 121-135.

[34] Q. D. Lã, Y. H. Chew, B.-H. Soong, Potential Game Theory, Springer, 2016.

[35] D. Lee, K. Huang, Passive-set-position-modulation framework for interactive robotic systems, IEEE Transactions on Robotics 26 (2) (2010) 354-369. 